\title{
PLÉIADES: RESPONSIVENESS, FLEXIBILITY, REACTIVITY
}

\author{
Ch. Gabriel-Robez ${ }^{\mathrm{a}^{*}}$, Rob Lees $^{\mathrm{b}}$, Marc Bernard ${ }^{\mathrm{a}^{*}}$ \\ a ASTRIUM ASV GEO, 5 rue des Satellites, BP14359, 31030 Toulouse, France. charlotte.gabriel-robez@ spotimage.fr ; \\ marc.bernard@spotimage.fr \\ ${ }^{\mathrm{b}}$ ASTRIUM ASV GEO, Spot Image Services, Canberra, Australia. lees@ spotimage.com.au
}

KEY WORDS: Pléiades, phased constellation, disaster, mapping, monitoring, reliability, reactivity

\begin{abstract}
:
By the end of 2011, Astrium GEO-Information Services launched Pléiades 1, the first of two identical optical imaging satellites that will be operated on a phased orbit. This satellite system, designed by the French Space Agency, CNES, based upon French Defense specifications, will provide $50-\mathrm{cm}$ products in record time. The overall aim of this paper is to describe the benefits of the innovative features of Pléiades 1 and its operations, so as to assess their combined potential in emergency situations, crisis recovery, regular monitoring or large area mapping. Specific care will be brought to describe the reactivity enabled by the system.
\end{abstract}

Based on real-life examples, the paper will lead the analysis on the two main components of the system.

On the one hand, the space segment will be presented through the following characteristics: revisit capacity, agility, acquisition capacity and acquisition scenarios (target, single-pass mosaics, stereo, tristereo, linear monitoring, persistent surveillance).

On the other hand, the flexibility of the ground segment will be assessed. The benefits of multiple tasking plans per day, direct tasking capacity, automated processing and on-line ordering and delivering will be illustrated, tested and qualified for applications requiring a high level of responsiveness and reactivity.

The presentation will end with a summary of the benefits of the space segment features and the flexibility of the ground segment, fine-tuned to answer both military and civilian / commercial needs. The analysis will be extended in the perspective of the second Pléiades' launch, highlighting the advantages of having two satellites operating on a phased orbit, affording a daily revisit anywhere on Earth, with very high resolution.

\section{INTRODUCTION}

On December $16^{\text {th }}, 2011$, Pléiades 1 was launched from Kourou, French Guyana. The Soyuz launcher lifted off at 02:02:30 UTC, and satellite separation occurred at 02:58 UTC over Perth, Australia. Solar panels deployed 30 seconds later.

Pléiades 2 will follow roughly one year after. The Pléiades twins are two very high resolution satellites delivering $50 \mathrm{~cm}$ ortho color products. Operated as a true constellation on a phased orbit, the Pléiades system will afford unprecedented reactivity, including urgent programming and direct tasking mode. The Pléiades constellation is able to image any point on the globe every day. Its massive acquisition capacity (1 million sq. km / day / sat), broad swath $(20 \mathrm{~km})$ and outstanding agility open new levels of performance. Catalogue, new acquisitions, subscription services, online monitoring,... Pléiades services were designed to stand just a click away from any user desktop wherever in the world.

In fact, Pléiades 1 is only the first born of a 4-children family. The upcoming launch of Pléiades 2 and Spot $6 \& 7$ will offer unique solutions combining broad coverage, daily revisit and very high resolution products and services. Four satellites over the next four years will guarantee continued success for users' projects.

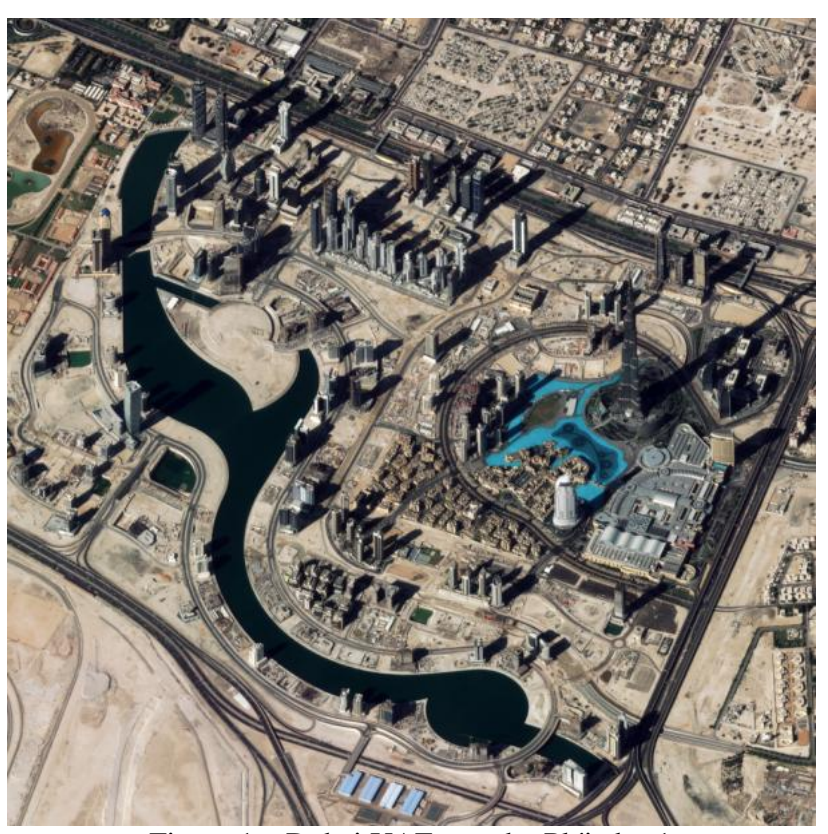

Figure 1 - Dubai UAE seen by Pléiades 1

Pansharpened product prototype from the commissioning phase. (c) CNES 2011 - distribution Astrium Services / Spot Image 


\section{AN ORIGINAL CONSTELLATION}

Indeed, with 3 new satellites set to launch until 2013 to complement Pléiades 1, all users can benefit from the very best that space technology has to offer. The 4 satellites will operate as a true constellation, combining a twice-daily revisit capability with an smart range of resolutions.

The twin Pléiades are a couple of very high resolution satellites delivering $50-\mathrm{cm}$ ortho color products as a standard. The two other brothers are SPOT-6 and 7, meant to extend SPOT-5's success in the $1.5 \mathrm{~m}$ product-resolution family.

Phased on the same orbit, the constellation will enjoy unprecedented reactivity, with intra-day revisit capacity anywhere on the Earth. Multiple tasking plans per day result in an unrivalled optimization of data collection: even chaotic weather changes as well as last-minute requests can be taken into account for a first-class level of service.

Furthermore, the constellation will bear an unheard-of complementarities. SPOT-6 and 7 are perfectly suited for any kind of applications requiring broad coverage (60 km swath), whereas Pléiades affords the largest swath in the submetric market (20 km at nadir), ensuring to all users a maximized coverage as well as easier data processing and handling.

\section{A MASSIVE COLLECTION CAPABILITY}

\subsection{Agility means Accessibility and Revisit}

Instead of "classical" gyros, the 4 satellites are equipped with CMG for state-of-the-art agility, enabling to fulfill more simultaneous requests in the same pass. The Pléiades satellites' gyros enable them to tilt very quickly along and across track to image different areas of interest.

Each satellite is able to collect imagery anywhere within an $800-\mathrm{km}$-wide ground strip, covering 200 kilometres in 11 seconds or 800 kilometres in 25 seconds, including stabilization time.

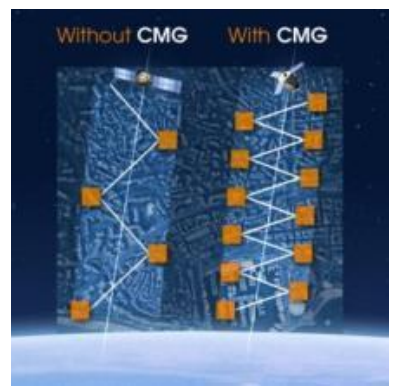

Figure 2 - Illustration of CMG's effect on collection capability

\subsection{Accessibility means Acquisition capacity}

With an acquisition capacity reaching more than 1 million square kilometers per day and per satellite for Pléiades, and simultaneously 2.5 million square kilometers for SPOT-6 \& 7 , the constellation will provide unprecedented capability of serving the needs of customers worldwide.

\subsection{Specific collection modes tailored for applications}

The agility thus allows imaging multiple targets (typically 15 targets over 1,000 km within a corridor of +/-30 degrees).

To answer mapping and monitoring needs over large areas, Pléiades is also able to collect large mosaics in a single pass, more than $100 \times 100 \mathrm{~km}$ from (within a $30^{\circ}$ incidence angle), as shown in Figure 3 below.

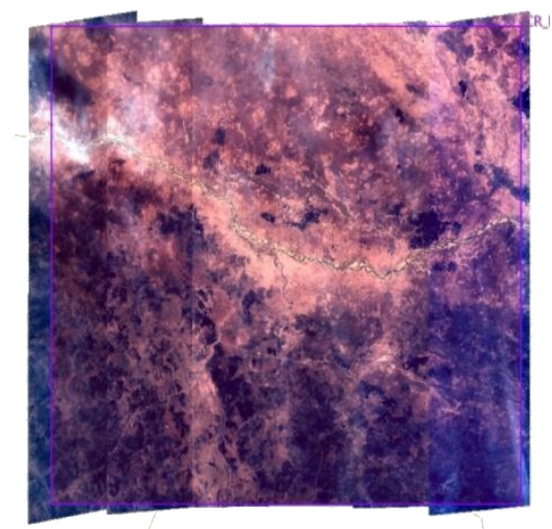

Figure 3 - Single-pass Pléiades collection imaging a $1^{\circ} \mathrm{x} 1^{\circ}$ geocell over Chad (purple frame) on Feb 10 ${ }^{\text {th }}, 2012$.

Both Pléiades and SPOT-6\&7 satellites can also collect stereo and tri-stereo data for 3D applications. Beyond this, multi-stereo is also on the menu: Pléiades 1 recently collected 17 images over the city of Melbourne within a single pass, thus enabling a new area of applications (monitoring of the speed of vehicles, true ortho processing,...).

For coastal, border or river drains surveillance, the "corridor" tasking mode can be activated, to minimize the collection time and ensure a simultaneous capture over all the AOI.

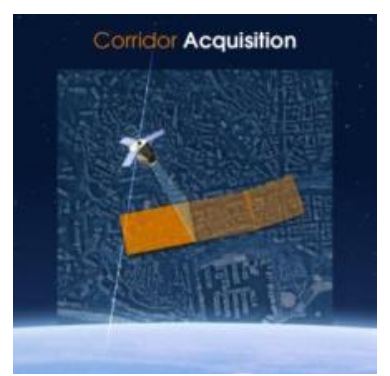

Figure 4 - Illustration of the "corridor mode"

For the user, this simply means a reduced average acquisition time window to collect his/her AOI, and a better homogeneity and a better consistency within adjacent images (through a larger simultaneity).

\section{REACTIVITY AT HAND}

\subsection{Reactivity also means increased efficiency}

The ground operations component is organized with maximum responsiveness in mind.

Pléiades work plans are uploaded to the satellite 3 times a day from 3 different points around the globe: the Kerguelen Islands for morning passes, the best time to upload tasking commands 
for Europe, Africa and the Middle East ; in Sweden for midday orbits and coverage of North and South America ; in Toulouse for evening passes over Asia and Oceania (Figure 5).

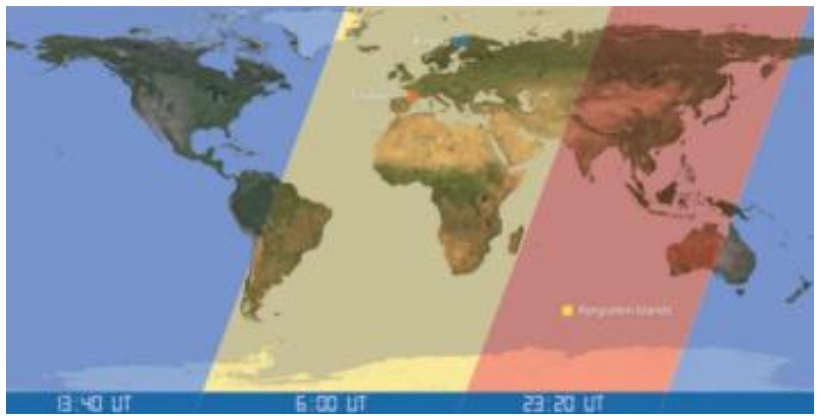

Figure 5: the 3 Pléiades' daily collection plans

This enables us to task users' requests up to two hours before satellite pass, thus reducing the lead time between tasking requests and image acquisition to a minimum.

It should be noted that the splitting into 3 parts of the daily collection plan also increases the global efficiency of the whole satellite system: using 3 different weather forecasts a day results into a more precise prediction of cloud position under the orbit, hence optimising the probability of getting cloud-free images.

\subsection{Direct tasking: Reactivity at customer's hand}

Pléiades customers with receiving stations configured for direct tasking will be able to refine tasking plans at the last moment (30 minutes prior to satellite arrival above the cone) according to the latest weather forecasts or emergency requests.

\section{GROUND PROCESSING: A SPEED-UP STORY}

A lot of work has gone into designing image production systems. The fully automatic orthorectification process is capable of generating a $20 \mathrm{~km} \times 20 \mathrm{~km}$ Pléiades colour image in less than 30 minutes and a single-pass mosaic of $60 \mathrm{~km}$ x $60 \mathrm{~km}$ in less than 3 hours.

On the user side, everything from ordering to data delivery has been made as flexible and easy as possible. New acquisitions, catalogue data, subscription offers, collection alerts, online monitoring services and more mean that imagery is just a click away, ready to use.

\section{CONCLUSION}

The consecutive launches of Pléiades-1 \& 2 and SPOT-6 \& 7 signal a paradigm shift in the world of Earth Observation services. We are now fully ready to operate a coherent, optimized multi-resolution constellation of 4 satellites that will deliver to the users a high level of performance: revisit, collection capability, responsiveness, accuracy...

Last but not least, this constellation will ensure continuity of service up to 2023, securing the users' investments into VHR data.

Acknowledgments: the authors would like to thank all the teams who contributed to the success of Pléiades during the conception, launch and calibration phases, especially our colleagues from CNES and ASTRIUM (satellite branch). 